\title{
Linear and nonlinear optical properties of erbium doped zinc borotellurite glass system
}

\begin{abstract}
A glass series of erbium doped zinc borotellurite glass system was prepared by using the melt-quenching method. The absorption spectra revealed several bands at visible range which correspond to the following transitions (from the ground state); 4G11/2 + 2H9/2 + 4F5/2 + $4 \mathrm{~F} 7 / 2+2 \mathrm{H} 11 / 2+4 \mathrm{~S} 3 / 2+4 \mathrm{~F} 9 / 2+4 \mathrm{I} 9 / 2+4 \mathrm{I} 11 / 2$. From the Juddï Ofelt analysis, it is found that the trend of $\mathrm{q} 2$ values is a non-linear variation along with erbium concentrations. Meanwhile, the value of $\mathrm{q} 6$ decreases as the erbium concentration increases. The photoluminescence analysis shows green emission which are attributed to the 4S3/2 level to the ground state at 4I15/2. Meanwhile, the upconversion analysis revealed several emission bands at $376 \mathrm{~nm}, 424 \mathrm{~nm}, 470 \mathrm{~nm}$ and $558 \mathrm{~nm}$ which correspond to 4G11/2 $\ddot{Y}$ 4I15/2, 4F3/2 $\ddot{Y}$ 4I15/2, 4F7/2 $\ddot{Y}$ 4I15/2 and 4S3/2 $\ddot{Y}$ 4I15/2 transitions respectively. The non-linear refractive index spectra show self-defocusing behavior and negative nonlinear refraction $(62<0)$ under laser excitation at $532 \mathrm{~nm}$ of wavelength. The obtained values of nonlinear absorption and nonlinear susceptibility revealed nonlinear variations.
\end{abstract}

Keyword: Borotellurite glass; Luminescence; Upconversion; Nonlinear optical properties 